\title{
Inflationary attractor in Gauss-Bonnet brane cosmology
}

\author{
Xin-He Meng ${ }^{1,2,3} * \quad$ Peng Wang ${ }^{1} \dagger$ \\ 1. Department of Physics, Nankai University, Tianjin 300071, P.R.China \\ 2. Institute of Theoretical Physics, CAS, Beijing 100080, P.R.China \\ 3. Department of Physics, University of Arizona, Tucson, AZ 85721
}

\begin{abstract}
The inflationary attractor properties of the canonical scalar field and Born-Infeld field are investigated in the Randall-Sundrum II scenario with a Gauss-Bonnet term in the bulk action. We find that the inflationary attractor property will always hold for both the canonical and Born-Infeld fields for any allowed non-negative Gauss-Bonnet coupling. We also briefly discuss the possibility of explaining the suppressed lower multiples and running scalar spectral index simultaneously in the scenario of Gauss-Bonnet brane inflation.
\end{abstract}

\section{Introduction}

Recently, there is considerable interest in inflationary models motivated by string/M-theory (See [1] for a review and reference therein). In particular, much attention has been focused on the braneworld scenario, where our observable four-dimensional universe is modelled as a domain wall embedded in a higher-dimensional bulk space 2]. An important realization of this picture is provided by the Randall-Sundrum type II scenario (RSII), where a spatially isotropic and homogenous brane propagates in a 5-dimensional bulk space with an effectively negative cosmological constant [3]. This scenario has many interesting cosmological implications. The standard Friedmann equation would be modified when the energy density of the matter confined to the brane is larger than the brane tension [4]. This Modified Friedmann equation will lead to a faster Hubble expansion and a more strongly damped evolution of the scalar field. This assists the slow-roll inflation, enhances the amount of inflation obtained in any given inflation model, and drives the perturbations towards an exactly scale-invariant Harrison-Zel'dovich spectrum. Inflation in RSII model has been studied for both canonical scalar field (see e.g. [5]) and Born-Infeld field (see e.g. [6]).

One approach to developing the braneworld scenario in a more string theoretic setting is to introduce higher-order curvature invariants in the bulk action [7, 8, 9, 11]. Specifically, the Gauss-Bonnet combination arises as the leading order for quantum corrections in the heterotic string effective action and in five dimensions it represents the unique combination of curvature invariants that leads to second-order field equations in the metric tensor [14]. Note that quantum effects on the brane also induce higher curvature terms [13], where, however, the Gauss-Bonnet term is topological invariant. In the RSII scenario with a Gauss-Bonnet term, the effective Friedmann equation describing the motion of brane containing general perfect fluid may be derived from a generalization of the Birkhoff's theorem [8]. A more geometrical approach may be taken by varying the boundary term [9] which was calculated by Myers [10] or employing the formalism of differential forms [11]. Braneworld holography in Gauss-Bonnet gravity was studied in Ref. 12], where it was demonstrated that a holographic description for braneworld matter could be found in the Gauss-Bonnet bulk.

Thus, the investigation of the effects of a Gauss-Bonnet term on inflationary braneworld models in the RSII scenario is well motivated. This was analyzed in detail first in Ref.[15], where the authors have derived the expressions for slow-roll parameters and spectral indices, etc. However, up to now, some features of Gauss-Bonnet brane inflation have not been investigated in detail in the literature (See Ref. [16] for a recent discussion). Specifically, if inflation is to be truly predictive, the evolution when the scalar field is at some given point on the potential has to be independent of the initial conditions. Otherwise, any result, such as the amplitude of density perturbations, would depend on the unknown initial conditions. However, the equation of motion for the scalar field is second order, implying that $\dot{\phi}$ can in principle take on any value anywhere on the potential provided, and so there certainly is not a unique solution at each point on the potential. Inflation can therefore only be predictive if the solution exhibits an attractor behavior, where the differences between solutions of different initial conditions rapidly vanish [17] (See Sec.3.7 of Ref. 18] for a review). Thus it is important to investigate whether the inflationary attractor property holds for Gauss-Bonnet brane inflation driven by the canonical scalar field or Born-Infeld field. In RSII scenario without the Gauss-Bonnet contribution, the inflationary attractor property is shown to be held for both the canonical scalar field and Born-Infeld field [20]. In the standard FRW cosmology, the inflationary attractor property is shown to be held for Born-Infeld field in Ref. 222]. In this paper, we will show that the attractor property still holds for both the canonical and Born-Infeld fields in the Gauss-Bonnet braneworld scenario. The conclusions we get in this paper is quite general and model-independent.

\section{Modified Friedmann equation in Gauss-Bonnet brane cosmology}

\footnotetext{
*xhmeng@phys.nankai.edu.cn

$\dagger$ pwang234@sohu.com
} 
In this section, we will follow closely Ref. [15]. The 5-dimensional bulk action for the Gauss-Bonnet braneworld scenario is given by

$$
S=\frac{1}{2 \kappa_{5}^{2}} \int_{\mathcal{M}} d^{5} x \sqrt{-g}\left[R-2 \Lambda+\alpha\left(R^{2}-4 R_{A B} R^{A B}+R_{A B C D} R^{A B C D}\right)\right]+S_{\partial \mathcal{M}}+S_{m}
$$

where $\alpha \geq 0$ with dimension (mass) $)^{2}$ is the Gauss-Bonnet coupling, $\Lambda<0$ is the bulk cosmological constant and $\kappa_{5}^{2}=8 \pi \bar{M}_{5}^{-3}$ determines the $5 \mathrm{~d}$ Planck scale. The $S_{\partial \mathcal{M}}$ is the boundary term that is required to cancel normal derivatives of the metric tensor which arises when varying the action with respect to the metric [10], and the $S_{m}$ is the action for matter confined on the brane. We assume a $\mathbf{Z}_{2}$ symmetry across the bane. We will consider the case that a perfect fluid matter source with density $\rho$ is confined to the brane and the brane have a tension $\lambda$.

Define a new constant, $b$ :

$$
b \equiv\left(1+\frac{4}{3} \alpha \Lambda\right)^{3 / 2}
$$

Note that from Eq.(2), since $\Lambda<0$, there is an upper bound on $\alpha$

$$
\alpha \leq \frac{3}{4|\Lambda|} \equiv \alpha_{u}
$$

In order that the standard Friedmann equation be recovered at sufficiently low energy scales $(\rho \ll \lambda)$, we have the identification

$$
\kappa_{4}^{2} \equiv \frac{8 \pi}{M_{4}^{2}}=\frac{\kappa_{5}^{4} \lambda}{6 b^{2 / 3}}
$$

where $M_{4}$ is the $4 d$ Planck scale.

Introducing a dimensionless variable $x$, the Modified Friedmann equations are

$$
\begin{gathered}
\rho+\lambda=\left(\frac{\lambda b^{1 / 3}}{3 \alpha \kappa_{4}^{2}}\right)^{1 / 2} \sinh x \\
H^{2}=\frac{1}{4 \alpha}\left[b^{1 / 3} \cosh \left(\frac{2 x}{3}\right)-1\right]
\end{gathered}
$$

We assume that during inflation, $\rho \gg \lambda$, since this is the case that the Modified Friedmann equation is different from the standard one. Thus we can replace Eq. (5) by

$$
\rho=\left(\frac{\lambda b^{1 / 3}}{3 \alpha \kappa_{4}^{2}}\right)^{1 / 2} \sinh x
$$

Note $\rho$ increases monotonically with $x$.

In order for that the $4 \mathrm{~d}$ effective cosmological constant vanishes, the brane tension should satisfy

$$
\lambda=\frac{3}{2} \frac{1-b^{1 / 3}}{\alpha \kappa_{4}^{2}}
$$

It is easy to see that $\lambda \sim|\Lambda| / \kappa_{4}^{2}$ for any $0 \leq \alpha \leq \alpha_{u}$.

\section{Attractor property for canonical scalar field}

We consider a canonical scalar field confined on the brane. It is described by the Lagrangian

$$
L_{\text {canonical }}=-\frac{1}{2}\left(\partial_{\mu} \phi\right)^{2}-V(\phi)
$$

where we use the metric signature $\{-,+,+,+\}$. In a spatially flat FRW universe, we can assume that $\phi$ is spatially homogeneous. The energy density and the pressure are given by

$$
\rho_{\phi}=\frac{1}{2} \dot{\phi}^{2}+V(\phi)
$$




$$
p_{\phi}=\frac{1}{2} \dot{\phi}^{2}-V(\phi)
$$

The evolution equation of the field $\phi$ is

$$
\ddot{\phi}+3 H \dot{\phi}+V^{\prime}(\phi)=0
$$

where a prime denotes differentiation with respect to $\phi$.

In the high energy limit, i.e. $\rho_{\phi} \gg \lambda$, Eq.(7) can be written as

$$
\frac{1}{2} \dot{\phi}^{2}+V(\phi)=\left(\frac{\lambda b^{1 / 3}}{3 \alpha \kappa_{4}^{2}}\right)^{1 / 2} \sinh x
$$

In analyzing the inflationary attractor property, we will use the Hamilton-Jacobi formulation of the Friedmann equation [19]. In this formulation, we will view the scalar field $\phi$ as the time variable. This requires that the $\phi$ field does not change sign during inflation. Without loss of generality, we can choose $\dot{\phi}>0$ in the following discussions.

Differentiating Eq. (6) with respect to $t$ gives

$$
H \dot{H}=\frac{b^{1 / 3}}{12 \alpha} \sinh \left(\frac{2 x}{3}\right) \dot{x}
$$

Differentiating Eq.(13) with respect to $t$ and using Eq. (12) give

$$
\dot{x}=-3 H\left(\frac{3 \alpha \kappa_{4}^{2}}{\lambda b^{1 / 3}}\right)^{1 / 2} \frac{\dot{\phi}^{2}}{\cosh x}
$$

Substituting Eq. 15) into Eq.(14), we have

$$
H^{\prime}(\phi)=-\frac{b^{1 / 3}}{4 \alpha}\left(\frac{3 \alpha \kappa_{4}^{2}}{\lambda b^{1 / 3}}\right)^{1 / 2} \frac{\sinh \frac{2 x}{3}}{\cosh x} \dot{\phi}
$$

Substituting this equation into Eq. (13) gives

$$
\frac{8 \alpha \lambda}{3 b^{1 / 3} \kappa_{4}^{2}}\left(\frac{\cosh x}{\sinh \frac{2 x}{3}}\right)^{2} H^{\prime}(\phi)^{2}+V(\phi)=\left(\frac{\lambda b^{1 / 3}}{3 \alpha \kappa_{4}^{2}}\right)^{1 / 2} \sinh x
$$

Eqs.(16), (17) and (6) are the Hamilton-Jacobi formulation of the Modified Friedmann equations, which is more convenient to be used in analyzing the inflationary attractor behaviors than Eqs. (12), (7) and (6). In this formulation, one considers $H(\phi)$, rather than $V(\phi)$, as the fundamental quantities. If we can solve $H(\phi)$ from Eqs.(16) and (6), substituting into Eqs.(17) and (6) we can immediately obtain $V(\phi)$. Therefore, the Hamilton-Jacobi formalism is also very useful to obtain a large set of exact inflationary solution (See Ref. 20] for some examples) and put general constraints on the form of the potentials [21].

Supposing $\left(H_{0}(\phi), x_{0}\right)$ is any solution to Eqs.(17) and (6), which can be either inflationary or non-inflationary. We will consider the homogeneous perturbations $(\delta H(\phi), \delta x)$ to this solution. The attractor property will be satisfied if it becomes small as $\phi$ increases. Substituting $H(\phi)=H_{0}(\phi)+\delta H(\phi)$ and $x=x_{0}+\delta x$ into Eqs. (17), (6) and linearizing, we find that the perturbations obey

$$
\begin{gathered}
\delta x=\frac{12 \alpha H_{0}(\phi)}{b^{1 / 3} \sinh \frac{2 x_{0}}{3}} \delta H(\phi) \\
H_{0}^{\prime}(\phi) \delta H^{\prime}(\phi)=g(\phi) \delta x
\end{gathered}
$$

where $g(\phi)$ is given by

$$
g(\phi)=\frac{3 b^{1 / 3} \kappa_{4}^{2}}{16 \alpha \lambda}\left(\frac{\lambda b^{1 / 3}}{3 \alpha \kappa_{4}^{2}}\right)^{1 / 2} \frac{\left(\sinh \frac{2 x_{0}}{3}\right)^{2}}{\cosh x_{0}}-\left(\tanh x_{0}-\frac{2}{3} \operatorname{coth} \frac{2 x_{0}}{3}\right) H_{0}^{\prime}(\phi)^{2}
$$


Eqs.(18), (19) can be integrated to give

$$
\delta H(\phi)=\delta H\left(\phi_{i}\right) \exp \left[\frac{12 \alpha}{b^{1 / 3} \sinh \frac{2 x_{0}}{3}} \int_{\phi_{i}}^{\phi} g(\phi) \frac{H_{0}(\phi)}{H_{0}^{\prime}(\phi)} d \phi\right]
$$

where $\delta H\left(\phi_{i}\right)$ is the value at some initial point $\phi_{i}$.

Since $H_{0}^{\prime}$ and $d \phi$ have the opposite sign, if $H_{0}$ is an inflationary solution, the perturbations will damp exponentially if $g(\phi)>0$, i.e.

$$
\frac{3 b^{1 / 3} \kappa_{4}^{2}}{16 \alpha \lambda}\left(\frac{\lambda b^{1 / 3}}{3 \alpha \kappa_{4}^{2}}\right)^{1 / 2} \frac{\left(\sinh \frac{2 x_{0}}{3}\right)^{2}}{\cosh x_{0}}-\left(\tanh x_{0}-\frac{2}{3} \operatorname{coth} \frac{2 x_{0}}{3}\right) H_{0}^{\prime}(\phi)^{2}>0
$$

Using Eqs. (12) and (16), this can be written as

$$
\operatorname{coth} x_{0} V(\phi)>\left(\tanh x_{0}-\frac{2}{3} \operatorname{coth} \frac{2 x_{0}}{3}-\frac{1}{2} \operatorname{coth} x_{0}\right) \dot{\phi}^{2}
$$

Since $\tanh x_{0}-\frac{2}{3} \operatorname{coth} \frac{2 x_{0}}{3}-\frac{1}{2} \operatorname{coth} x_{0}<0$ for all $x_{0}>0$, this inequality is always satisfied. Thus we conclude that the inflationary attractor property will hold for canonical scalar field for any $0 \leq \alpha \leq \alpha_{u}$.

Remark: If considering the phantom inflation [27, 30], it has been shown in Ref. [27] that in the RSII scenario the inflationary attractor property does not hold. It can be shown by almost the same derivation of this section that the inflationary attractor still does not hold in the Gauss-Bonnet braneworld for any $\alpha$. Thus, from the point of view of the inflationary attractor property, the scenario of phantom inflation is unappealing.

\section{Attractor property for Born-Infeld field}

The effective Lagrangian for tachyon is the Born-Infeld action [23, 24]

$$
L_{B I}=-V(\phi) \sqrt{1+\left(\partial_{\mu} \phi\right)^{2}}
$$

In a spatially flat FRW universe, we can assume that $\phi$ is spatially homogeneous. The energy density and the pressure are given by

$$
\begin{gathered}
\rho_{\phi}=\frac{V(\phi)}{\sqrt{1-\dot{\phi}^{2}}} \\
p_{\phi}=-V(\phi) \sqrt{1-\dot{\phi}^{2}}
\end{gathered}
$$

The evolution equation of $\phi$ is

$$
\frac{\ddot{\phi}}{1-\dot{\phi}^{2}}+3 H \dot{\phi}+\frac{V^{\prime}(\phi)}{V(\phi)}=0
$$

In the high energy limit, i.e. $\rho_{\phi} \gg \lambda$, Eq.(7) can be written as

$$
\frac{V(\phi)}{\sqrt{1-\dot{\phi}^{2}}}=\left(\frac{\lambda b^{1 / 3}}{3 \alpha \kappa_{4}^{2}}\right)^{1 / 2} \sinh x
$$

Differentiating Eq.(28) with respect to $t$ and using Eq.(27) give

$$
\dot{x}=-3 H\left(\frac{3 \alpha \kappa_{4}^{2}}{\lambda b^{1 / 3}}\right)^{1 / 2} \frac{V(\phi) \dot{\phi}^{2}}{\cosh x \sqrt{1-\dot{\phi}^{2}}}
$$

Substituting Eq.(29) into Eq.(14) gives

$$
H^{\prime}(\phi)=-\frac{b^{1 / 3}}{4 \alpha}\left(\frac{3 \alpha \kappa_{4}^{2}}{\lambda b^{1 / 3}}\right)^{1 / 2} \frac{\sinh \frac{2 x}{3}}{\cosh x} \frac{V(\phi) \dot{\phi}}{\sqrt{1-\dot{\phi}^{2}}}
$$


Substituting this equation into Eq.(28), we can get

$$
\frac{16 \alpha \lambda}{3 b^{1 / 3} \kappa_{4}^{2}}\left(\frac{\cosh x}{\sinh \frac{2 x}{3}}\right)^{2} H^{\prime}(\phi)^{2}+V(\phi)^{2}=\frac{\lambda b^{1 / 3}}{3 \alpha \kappa_{4}^{2}} \sinh ^{2} x
$$

Eqs. (30), (31) and (6) are the Hamilton-Jacobi formulation of the Modified Friedmann equations.

As in the previous section, assuming $\left(H_{0}(\phi), x_{0}\right)$ is any solution to Eqs. (31) and (6), substituting $H(\phi)=H_{0}(\phi)+$ $\delta H(\phi)$ and $x=x_{0}+\delta x$ into Eqs.(31), (6) and linearizing, we find that the perturbations obey

$$
\begin{gathered}
\delta x=\frac{12 \alpha H_{0}(\phi)}{b^{1 / 3} \sinh \frac{2 x_{0}}{3}} \delta H(\phi) \\
H_{0}^{\prime}(\phi) \delta H^{\prime}(\phi)=h(\phi) \delta x
\end{gathered}
$$

where $h(\phi)$ is given by

$$
h(\phi)=\frac{b^{2 / 3}}{16 \alpha^{2}} \frac{\sinh x_{0} \sinh ^{2} \frac{2 x_{0}}{3}}{\cosh x_{0}}-\left(\tanh x_{0}-\frac{2}{3} \operatorname{coth} \frac{2 x_{0}}{3}\right) H_{0}^{\prime}(\phi)^{2}
$$

Eqs. (32), can be integrated to give

$$
\delta H(\phi)=\delta H\left(\phi_{i}\right) \exp \left[\frac{12 \alpha}{b^{1 / 3} \sinh \frac{2 x_{0}}{3}} \int_{\phi_{i}}^{\phi} h(\phi) \frac{H_{0}(\phi)}{H_{0}^{\prime}(\phi)} d \phi\right]
$$

where $\delta H\left(\phi_{i}\right)$ is the value at some initial point $\phi_{i}$.

Since $H_{0}^{\prime}$ and $d \phi$ have the opposite sign, if $H_{0}$ is an inflationary solution, the perturbations will damp exponentially if $h(\phi)>0$, i.e.

$$
\frac{b^{2 / 3}}{16 \alpha^{2}} \frac{\sinh x_{0} \sinh ^{2} \frac{2 x_{0}}{3}}{\cosh x_{0}}-\left(\tanh x_{0}-\frac{2}{3} \operatorname{coth} \frac{2 x_{0}}{3}\right) H_{0}^{\prime}(\phi)^{2}>0
$$

Using Eqs.(28), (30), this can be written as

$$
\operatorname{coth} x_{0}>\left(\tanh x_{0}-\frac{2}{3} \operatorname{coth} \frac{2 x_{0}}{3}\right) \dot{\phi}^{2}
$$

Note from the action (24), $\dot{\phi}^{2} \leq 1$. In presence of this constraint on $\dot{\phi}^{2}$, it is easy to check that inequality (37) is always satisfied. Thus the inflationary attractor property will hold for Born-Infeld tachyon for any $0 \leq \alpha \leq \alpha_{u}$.

Remark: If considering the Born-Infeld phantom field [26], it has been shown in Ref. 27] that in the RSII scenario the inflationary attractor property does not hold. It can be shown by almost the same derivation of this section that the condition for the inflationary attractor property to hold for Born-Infeld phantom is $\alpha_{c} \leq \alpha \leq \alpha_{u}$ where $\alpha_{c} \sim \frac{\lambda}{\kappa_{4}^{2} \rho_{\phi}^{2}}$. Thus it is interesting to see that the presence of a Gauss-Bonnet term in the bulk action can restore the inflationary attractor property of Born-Infeld phantom.

\section{Conclusions and discussions}

In this paper, we have derived the Hamilton-Jacobi equations of inflation in the Randall-Sundrum II scenario with a Gauss-Bonnet contribution in the bulk action driven by a canonical or Born-Infeld scalar fields. Using those equations, we studied the inflationary attractor properties of the canonical and Born-Infeld scalar fields. We found that the inflationary attractor property holds for both the canonical and Born-Infeld fields for all the allowed nonnegative Gauss-Bonnet coupling constant. This justifies that it is sensible to discuss Gauss-Bonnet inflation driven by either canonical or Born-Infeld field without a fine-tuning of the initial conditions of the inflaton field in order that the inflation happens.

Finally, we will present an observation of an interesting feature of the MF equations (7) and (6), which makes the scenario of inflation in Gauss-Bonnet brane cosmology rather appealing: At sufficiently high energies, i.e. $x \gg 1$, the MF equations can be approximated by $H^{2} \propto \rho^{2 / 3}$. At low energies, i.e. $x \ll 1$, the MF equations will behave like $H^{2} \propto \rho^{2}$. In either case, the MF equation behaves like $H^{2}=A \rho^{q}$, while $q$ will increase when the energy decreases [15]. For this form of Friedmann equation, the amplitude of density perturbations is given by

$$
A_{s}^{2}=\frac{9 A^{3} V^{3 q}}{25 \pi^{2} V^{\prime 2}}
$$


The scalar spectral index is given by

$$
n_{s}-1=\frac{1}{A V^{q-1}}\left(\frac{2 V^{\prime \prime}}{3 V}-\frac{q V^{\prime 2}}{V^{2}}\right)
$$

Then, at high energy region, i.e. large scales, where $q=2 / 3<1$, the amplitude of density perturbations will be suppressed relative to the standard Friedmann evolution. Furthermore, when energy scale decreases, i.e. $q$ increases, the spectral index will decrease, i.e. the running is negative. Thus, the two important features of CMB observations by WMAP [28] may be explained simultaneously in the framework of Gauss-Bonnet brane cosmology without a changing of the potentials or tuning of the initial conditions [29]. Here, those features are due to modification of cosmic dynamics in early universe! This is a rather elegant possibility. In this work, we have focused on the modelindependent features of the Gauss-Bonnet brane inflation. Next, it is interesting to see whether some specific model can accommodate the data easily.

\section{Acknowledgements}

We would like to thank S.D.Odintsov and S.Nojiri for valuable comments and suggestions on the first draft of the manuscript. We would like to thank I.P.Neupane for valuable comments on the first uploaded version of this paper. We would also like to thank D.Lyth and N.J.Nunes for helpful discussions. This work is partly supported by Doctoral Foundation of National Education Ministry and ICSC-World Laboratory Scholarship.

[1] J.E.Lidsey, astro-ph/0305528 J.E.Lidsey, D.Wands and E.J.Copeland, Phys.Rep. 337 (2000) 343; M.Gasperini and G.Veneziano, Phys.Rep. 373 (2003) 1; M.Quevedo, Class.Quant.Grav. 19 (2002) 5721 hep-th/0210292;

[2] N.Arkani-Hamed, S.Dimopoulos and G.Dvali, Phys.Lett. 429 (1998) 263; I.Antoniadis, N.Arkani-Hamed, S.Dimopoulos and G.Dvali, Phys.Lett. 436 (1998) 257; L.Randall and R.Sundrum, Phys.Rev.Lett. 83 (1999) 3370;

[3] L.Randall and R.Sundrum, Phys.Rev.Lett. 83 (1999) 4690;

[4] P.Binetruy, C.Deffayet, U.Ellwanger and D.Langlois, Phys.Lett. B477 (2000) 285 hep-ph/9910219;

[5] R.Maartens, D.Wands, B.A.Bassett and I.P.C.Heard, Phys.Rev. D62 (2000) 041301 hep-ph/9912464; E.J.Copeland, A.R.Liddle and J.E.Lidsey, Phys.Rev. D64 (2001) 023509;

[6] M.C.Bento, O.Bertolami and A.A.Sen, Phys.Rev. D67 (2003) 063511 hep-ph/0208124]; S. Mukohyama, Phys.Rev. D66 (2002) 024009 hep-th/0204084;

[7] S.Nojiri and S.D.Odintsov, JHEP 0007 (2000) 049; S.Nojiri, S.D.Odintsov and S.Ogushi, Int.J.Mod.Phys. A16 (2001) 5085; S.Nojiri, S.D.Odintsov and S.Ogushi, Phys.Rev. D65 (2002) 023521; J.E.Lidsey, S.Nojiri and S.D.Odintsov, JHEP 06 (2002) 026; J.E.Kim, B.Kyae and H.M.Lee, Phys.Rev. D62 (2000) 045013; I.P.Neupane, JHEP 0009 (2000) 040 hep-th/0008190; I.P.Neupane, Phys.Lett. B512 (2001) 137 hep-th/0104226; Y. M. Cho, I.P. Neupane and P. S. Wesson, Nucl.Phys. B621 (2002) 388 hep-th/0104227; I.P.Neupane, Class.Quant.Grav. 19 (2002) 5507 hep-th/0106100; Y. M. Cho, I. P. Neupane, Int.J.Mod.Phys. A18 (2003) 2703 hep-th/0112227;

[8] C.Charmousis and J.Dufaux, Class.Quant.Grav. 19 (2002) 4671 hep-th/0202107;

[9] S.C.Davis, Phys.Rev. D67 (2003) 024030 hep-th/0208205;

[10] R.C.Myers, Phys.Rev. D36 (1987) 392;

[11] E.Gravanis and S.Willison, Phys.Lett. B562 (2003) 118 hep-th/0209076;

[12] J. P. Gregory and A. Padilla, Class.Quant.Grav. 20 (2003) 4221 hep-th/0304250;

[13] S.Nojiri and S.D.Odintsov, Phys.Lett. 484 (2000) 119 hep-th/0006232;

[14] B.Zweibach, Phys.Lett. B156 (1985) 315; D.Lovelock, J.Math.Phys. 12 (1971) 498;

[15] J.E.Lidsey and N.J.Nunes, Phys.Rev. D67 (2003) 103510 astro-ph/0303168;

[16] B.C. Paul and M. Sami, hep-th/0312081

[17] D.S.Goldwirth, Phys.Lett. B243 (1990) 41; R.Brandenberger, G.Geshnizjani and S.Watson, Phys.Rev. D67 (2003) 123510 hep-th/0302222;

[18] A.R.Lidde and D.H.Lyth, Cosmological Inflation and Large Scale Structure, Cambrigde University Press, 2000;

[19] D.S.Salopek and J.R.Bond, Phys.Rev. D42 (1990) 3936; A.G.Muslimov, Class.Quant.Grav. 7 (1990) 231; J.E.Lidsey, Phys.Lett. B273 (1991) 42; A.R.Liddle, P.Parsons and J.D.Barrow, Phys.Rev. D50 (1994) 7222 astro-ph/9408015;

[20] Z.K.Guo, H.S.Zhang and Y.Z.Zhang, hep-ph/0309163.

[21] A.Vallinotto, E.J.Copeland, E.W.Kolb and A.R.Liddle, astro-ph/0311005

[22] Z.-K. Guo, Y.-S. Piao, R.-G. Cai and Y.-Z. Zhang, Phys.Rev. D68 (2003) 043508;

[23] M.R.Garousi, Nucl.Phys. B584 (2000) 284-299 hep-th/0003122;

[24] A.Sen, JHEP 0204 (2002) 048; ibid, JHEP 0207 (2002) 065;

[25] S.M.Carroll and M.Kaplinghat, Phys.Rev. D65 (2002) 063507 astro-ph/0108002;

[26] J.G.Hao and X.Z.Li, Phys.Rev. D68 (2003) 043501 hep-th/0305207; D.J.Liu and X.Z.Li, Phys.Rev. D68 (2003) 067301 hep-th/0307239;

[27] X.H.Meng and P.Wang, hep-ph/0311070

[28] D.N.Spergel, et al., Astrophys.J.Suppl. 148 (2003) 1 astro-ph/0302207 ; L.Page et al. astro-ph/0302220 M.Nolta, et al, astro-ph/0305097 C.Bennett, et al, Astrophys.J.Suppl. 148 (2003) 175 astro-ph/0302209; 
[29] C.R.Contaldi, M.Peloso, L.Kofman and A.Linde, JCAP 0307 (2003) 002 astro-ph/0303636; J. M. Cline, P. Crotty, J. Lesgourgues, JCAP 0309 (2003) 010 astro-ph/0304558;

[30] Y.-S. Piao and Y.-Z. Zhang, astro-ph/0401231 